\title{
Рациональное проектирование термоэлектрического охлаждающего устройства для переменных температурных условий эксплуатации
}

\author{
Ю. И. Журавлев \\ Национальный университет «Одесская морская академия», ул. Дидрихсона, 8, г. Одесса, 65029, Украина
}

\begin{abstract}
Рассмотрена возможность рационального проектирования термоэлектрического устройства в составе радиоэлектронной аппаратуры при эксплуатации в различных климатических условиях. Показана возможность выбора в качестве начального - наиболее нагруженного токового режима работь охлаждающего термоэлемента, обеспечивающего повымение показателей надежности при приемлемых энергетических затратах и массогабаритных характеристиках однокаскадного термоэлектрического устройства в проиессе изменения температурных условий окружающей среды.
\end{abstract}

Ключевье слова: Режим работы; Холодопроизводительность; Интенсивность отказов.

\section{Раціональне проектування термоелектричного охолоджуючого пристрою для змінних температурних умов експлуатації}

\section{Ю. І. Журавльов}

Національний університет «Одеська морська академія», вул. Дідріхсона, 8, м. Одеса, 65029, Україна

\begin{abstract}
Розглянуто можливість раціонального проектування термоелектричного пристрою в складі радіоелектронної апаратури при експлуатаиії в різних кліматичних умовах. Показана можливість вибору в якості початкового - найбільш навантаженого токового режиму роботи охолоджуючого термоелемента, шэо забезпечує підвищення показників надійності при прийнятних енергетичних витратах і масогабаритних характеристиках однокаскадного термоелектричного пристрою в процесі зміни температурних умов навколишнього середовища.
\end{abstract}

Ключові слова: Режим роботи; Холодопродуктивність; Інтенсивність відмов.

(C) The Author(s) 2017. This article is an open access publication

This work is licensed under the Creative Commons Attribution 4.0 International License (CC BY) http://creativecommons.org/licenses/by/4.0/

\section{1 Введение}

Процесс совершенствования радиоэлектронной аппаратуры (РЭА) характеризуется увеличением ее сложности, высокой плотностью компоновки и стабильным ростом степени интеграции элементной базы. При высокой плотности компоновки радиоэлектронная аппаратура должна обладать достаточной многофункциональностью, быстродействием, высокими показателями надежности при минимально возможных массогабаритных и энергетических характеристиках. Однако процесс одновременно приводит и к негативным последствиям: к увеличению плотности тепловых потоков, перегреву теплонагруженных элементов и их отказу.

Совершенствование и автоматизация технологических процессов производства пленочных элементов позволила уменьшить интенсивность их отказов примерно на два порядка. Однако с ростом температуры интенсивность отказов этих элементов резко увеличивается. Известно, что в такого рода приборах и элементах электроники от 40 до 90\% подводимой мощности превращается в тепло, которое необходимо отводить системами обеспечения тепловых режимов (СОТР) [1].

В ряде случаев необходимые параметры элементов РЭА могут быть получены при сравнительно низких и даже криогенных температурах. В этих условиях становится актуальным поиск и технологическая реализация эффективных способов обеспечения тепловых режимов РЭА с учетом ее конструктивных особенностей и условий эксплуатации. Сложность обусловлена еще тем, что РЭА эксплуатируются в условиях воздействия множества дестабилизирующих факторов. Одним из основных таких факторов, резко снижающих надежность РЭА, являются климатические воздействия при значительном диапазоне изменения температуры окружающей среды и условий теплообмена на внешней поверхности элементов и радиоэлектронной аппаратуры в целом.

Опыт разработки СОТР для РЭА позволяет выбрать в качестве наиболее рациональных и перспективных для термостабилизации РЭА следующие способы охлаждения:

- принудительное воздушное охлаждение; 
- принудительное жидкостное охлаждение;

- термоэлектрическое охлаждение;

- криогенное охлаждение.

Целью настоящей работы является поиск оптимальных конструктивных решений позволяющих при изменяющейся температуре среды выбрать вариант конструкции ТЭУ, обеспечивающий уменьшение энергетических затрат и повышение показателей надежности.

\section{2 Расчет параметров и показателей надежности TЭY}

Рассмотрим влияние температуры окружающей среды $T_{\mathrm{c}}$ на основные параметры и показатели надежности РЭА с использованием в качестве заданной одноканальной конструкции ТЭУ, рассчитанной на начальный наиболее тяжелый тепловой режим, с последующим переходом на другие токовые режимы работы.

Расчеты основных параметров и показателей надежности ТЭУ для различных начальных токовых режимов работы $\left(Q_{0 \max },\left(Q_{0} / I\right)_{\max },\left(Q_{0} / I^{2}\right)_{\max },-\lambda_{\min }\right)$, peзультаты которых приведены в таблице 1 , выполнялись в соответствии с приведенными в [2, с. 30-35] формулами, при следующих исходных данных:

- температура термостабилизации рабочего спая $T_{0}=293 \mathrm{~K}\left(+20^{\circ} \mathrm{C}\right)$;
- величина собственных тепловыделений объекта охлаждения $q_{0}=1,0 \mathrm{BT}$;

- температуры среды изменяется в пределах $T_{\mathrm{c}}=$ $=293-323 \mathrm{~K}$;

- температура тепловыделяющего спая $T=T_{\mathrm{c}}+10 \mathrm{~K}$.

Проведем расчет теплоизоляционного кожуха для объекта охлаждения по известным формулам $[3$, c. $72-89]$.

Количество тепловых потерь можно определить из соотношения

где $k=\left(1 / \alpha_{1}+\delta / \lambda+1 / \alpha_{2}\right)^{-1}$

$F$ - площадь поверхности теплоизоляционного кожуха;

$\Delta t=T_{\mathrm{c}}-T_{0} ;$

$\alpha_{1}-$ коэффициент теплопередачи внутри корпуса РЭА;

$\alpha_{2}$ - коэффициент теплопередачи внутри теплоизоляционного корпуса;

$\delta$ - толщина теплоизоляционного корпуса;

$\lambda_{\text {и }}-$ коэффициент теплопроводности теплоизоляции.

Общая тепловая нагрузка на объект охлаждения составляет

$$
Q_{0}=Q_{\mathrm{H}}+q_{0}
$$

Таблица 1 - Результаты расчета основных параметров и показателей надежности ТЭУ, полученные при $q_{0}=1,0$ Вт; $T_{0}=293 \mathrm{~K}\left(20^{\circ} \mathrm{C}\right) ; \quad T=T_{\mathrm{c}}+10 \mathrm{~K} ; \quad \alpha_{1}=20 \mathrm{BT} /\left(\mathrm{M}^{2} \cdot \mathrm{K}\right) ; \quad \alpha_{2}=10 \mathrm{BT} /\left(\mathrm{м}^{2} \cdot \mathrm{K}\right) ; \quad F=20 \mathrm{~cm}^{2} ; \quad \delta=5 \mathrm{Mм} ; \quad \lambda_{\text {и }}=41 \cdot 10^{-3} \mathrm{BT} /(\mathrm{M} \cdot \mathrm{K}) ;$ $t=10^{4} \mathrm{q} ; \quad l / S=10 \mathrm{~cm}^{-1}$ для различных токовых режимов работы

\begin{tabular}{|c|c|c|c|c|c|c|c|c|c|c|c|c|c|c|c|}
\hline $\begin{array}{l}T_{\mathrm{c}}, \mathbf{K} \\
\left({ }^{\circ} \mathrm{C}\right)\end{array}$ & $\begin{array}{l}T, \\
\mathbf{K}\end{array}$ & $\begin{array}{c}R \cdot 10^{3} \\
\text { OM }\end{array}$ & $I_{\max }, \mathrm{A}$ & B & $\begin{array}{c}\Delta T_{\max } \\
\mathbf{K}\end{array}$ & $\boldsymbol{\Theta}$ & $Q_{0}, \mathrm{BT}$ & I, & $\begin{array}{l}W \\
\mathbf{B T}\end{array}$ & $\begin{array}{l}\text { U, } \\
\mathbf{B}\end{array}$ & $\boldsymbol{E}$ & $\lambda / \lambda_{0}$ & $\begin{array}{c}\lambda \cdot 10^{8} \\
1 / 4\end{array}$ & $\begin{array}{l}\alpha F_{\mathrm{p}} \\
\mathbf{B T} / \mathbf{K}\end{array}$ & $P$ \\
\hline \multicolumn{16}{|c|}{$\begin{array}{l}\text { I вариант: начальный режим } Q_{0 \max } \\
n_{1}=8,2 \text { шт.; } B=1\end{array}$} \\
\hline $323(50)$ & 333 & 11,4 & 5,22 & 1,0 & 102,6 & 0,39 & 1,55 & 5,22 & 5,8 & 1,11 & 0,21 & 8,2 & 24,6 &, 74 & 0,99754 \\
\hline $313(40)$ & 323 & 11,1 & 5,33 & 0,58 & 103,0 & 0,291 & 1,37 & 3,1 & 2,05 & 0,65 & 0,67 & 0,87 & 2,6 &, 34 & 0,99974 \\
\hline $303(30)$ & 313 & 11,0 & 5,33 & 0,412 & 103,0 & 0,194 & 1,18 & 2,2 & 1,0 & 0,46 &, 18 & 0,18 & 0,551 &, 22 & 0,999945 \\
\hline $293(20)$ & 303 & 11,1 & 5,33 & 0,29 & 103,0 & 0,10 & 1,0 & 1,52 & 0,47 & 0,31 & 2,10 & 0,032 & 0,095 & 0,15 & 0,999991 \\
\hline \multicolumn{16}{|c|}{$\begin{array}{l}\text { II вариант: начальный режим }\left(Q_{0} / I\right)_{\max } \\
\qquad n=10,6 ; B=\Theta^{1 / 2}\end{array}$} \\
\hline $323(50)$ & 333 & 11,4 & 5,22 & 0,624 & 102,6 & 0,39 & 1,55 & 3,3 & 3,13 & 0,96 & 0,50 & 1,59 & 4,77 & 0,47 & 0,99952 \\
\hline $313(40)$ & 323 & 11,1 & 5,33 & 0,453 & 103,0 & 0,291 & 1,37 & 2,41 & 1,68 & 0,70 & 82 & 0,39 & 1,16 & 0,31 & 0,99988 \\
\hline $303(30)$ & 313 & 11,0 & 5,33 & 0,33 & 103,0 & 0,194 & 1,18 & 1,76 & 0,87 & 0,49 & 1,36 & 0,087 & 0,26 & 0,21 & 0,999974 \\
\hline $293(20)$ & 303 & 11,1 & 5,33 & 0,23 & 103,0 & 0,10 & 1,0 & 1,2 & 0,40 & 0,33 & 2,53 & 0,014 & 0,043 & 0,14 & 0,9999957 \\
\hline \multicolumn{16}{|c|}{$\begin{array}{l}\text { III вариант: начальный режим }\left(Q_{0} / I^{2}\right)_{\max }\left(E_{\max }\right) \\
n=21,0 \text { шт.; } B=\Theta\end{array}$} \\
\hline $323(50)$ & 333 & 11,4 & 5,22 & 0,39 & 102,6 & 0,39 & 1,55 & 2,0 & 2,68 & 1,34 & 0,58 & 0,43 & 1,3 & 0,42 & 0,99987 \\
\hline $313(40)$ & 323 & 11,1 & 5,33 & 0,29 & 103,0 & 0,291 & 1,37 & 1,55 & 1,51 & 0,97 & 0,91 & 0,11 & 0,34 & 0,29 & 0,999966 \\
\hline $303(30)$ & 313 & 11,0 & 5,33 & 0,21 & 103,0 & 0,194 & 1,18 & 1,11 & 0,76 & 0,68 & 1,55 & 0,023 & 0,07 & 0,19 & 0,999993 \\
\hline $293(20)$ & 303 & 11,0 & 5,33 & 0,14 & 103,0 & 0,10 & 1,0 & 0,71 & 0,30 & 0,43 & 3,3 & 0,003 & 0,0081 & 0,13 & 0,9999992 \\
\hline \multicolumn{16}{|c|}{$\begin{array}{l}\text { IV вариант: начальный режим } \lambda_{\min } \\
\qquad n=47,1 \text { шт; } B=\eta \Theta\end{array}$} \\
\hline $323(50)$ & 333 & 11,4 & 5,22 & 0,29 & 102,6 & 0,39 & 1,55 & 1,5 & 3,62 & 2,41 & 0,43 & 0,28 & 0,83 & 0,52 & 0,99997 \\
\hline $313(40)$ & 323 & 11,1 & 5,33 & 0,215 & 103,0 & 0,291 & 1,37 & 1,15 & 2,02 & 1,76 & 0,68 & 0,069 & 0,21 & 0,34 & 0,999979 \\
\hline $303(30)$ & 313 & 11,0 & 5,33 & 0,15 & 103,0 & 0,194 & 1,18 & 079 & 0,94 & 1,19 & 1,25 & 0,012 & 0,035 & 0,21 & 0,999996 \\
\hline $293(20)$ & 303 & 11,0 & 5,33 & 0,088 & 103,0 & 0,10 & 1,0 & 0,47 & 0,32 & 0,68 & 3,13 & 0,0009 & 0,0026 & 0,13 & 0,9999997 \\
\hline
\end{tabular}




\section{3 Анализ результатов моделирования однокас- кадного ТэУ}

На начальной стадии проектирования рассчитываем конструкцию ТЭУ для наиболее тяжелых температурных условий при $T_{\mathrm{c}}=323 \mathrm{~K}$ для различных токовых режимов работы [3]. С ростом температуры среды $T_{\mathrm{c}}$ для различных начальных токовых режимов работы:

- тепловая нагрузка увеличивается (рисунок 1);

- увеличивается относительный рабочий ток $B$, a, следовательно, и рабочий ток I (рисунок 2);

- увеличивается мощность потребления $W$ (рисунок 3, a), при этом она минимальна в режиме $\left(Q_{0} / I^{2}\right)_{\max }$ и составляет $2,7 \mathrm{BT}$, максимальна в режиме $Q_{0 \max }$ (при температуре среды, например, $T_{\mathrm{c}}=323 \mathrm{~K}$ значение $W$ в режиме $Q_{0 \max }$ в 2,2 раза больше, чем в режиме $\left(Q_{0} / I^{2}\right)_{\max }$, в 1,85 раза больше, чем в режиме $\left(Q_{0} / I\right)_{\max }$, и в 1,6 раза больше, чем в режиме $\left.\lambda_{\min }\right)$;

- увеличивается интенсивность отказов $\lambda / \lambda_{0}$ (рисунок 3, б);

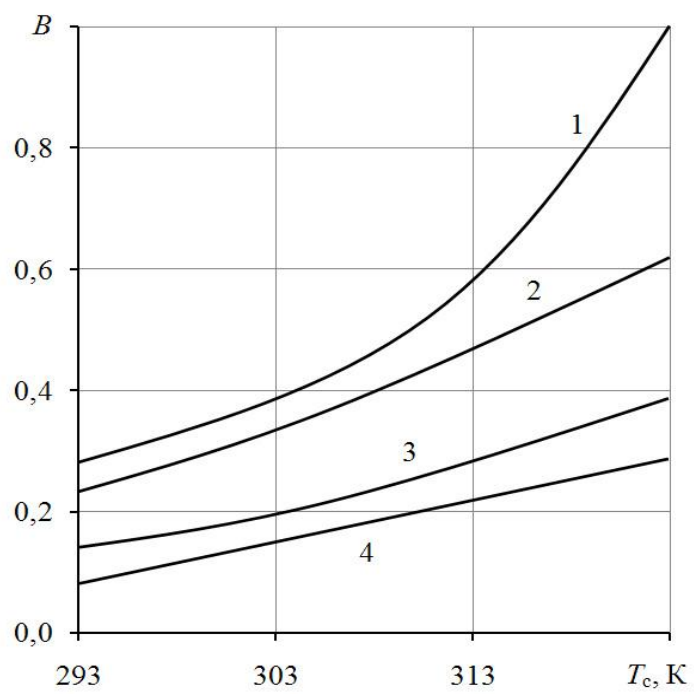

a)

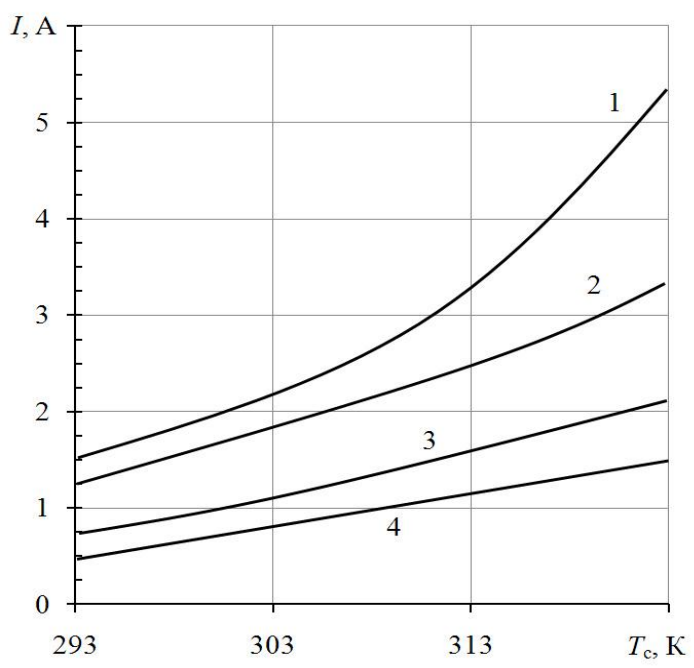

б)

Рисунок 2 - Зависимость относительного рабочего тока B (а) и рабочего тока I (б) однокаскадного термоэлектрического устройства от температуры среды $T_{c}$ nрu $T_{0}=293 \mathrm{~K} ; \mathrm{T}=T_{c}+10 \mathrm{~K} ; q_{0}=1,0 \mathrm{Bm} ; \mathrm{l} / \mathrm{S}=$ $=10 \mathrm{~cm}^{-1}$ для различных начальных токовых режимов работы: $1-Q_{\text {оmax }}(n=8,2) ; 2-\left(Q_{0} / I\right)_{\max }(n=10,6)$; $3-\left(Q_{0} / I^{2}\right)_{\text {max }}(n=21,0) ; 4-\lambda_{\text {min }}(n=47,1)$
- увеличивается тепловыводящая способность радиатора $\alpha F_{\mathrm{p}}$ (рисунок 4).

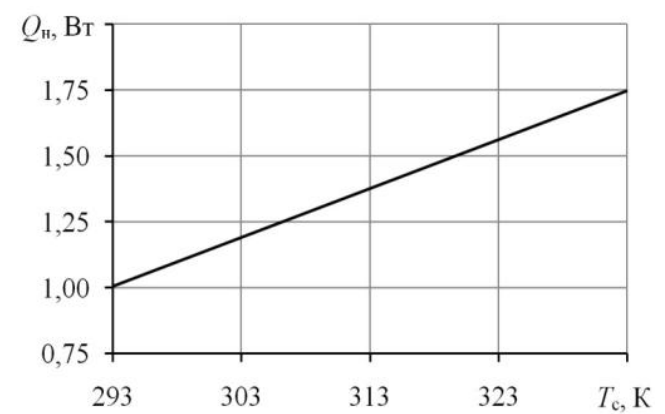

Рисунок 1 - Зависимость тепловой нагрузки $Q_{н}$ ТЭУ от температуры среды $T_{c}$ при $T_{0}=293 \mathrm{~K}$;

$T=T_{c}+10 \mathrm{~K} ; q_{0}=1,0 \mathrm{Bm} ; \alpha_{1}=20 \mathrm{Bm} /\left(\mathrm{M}^{2} \cdot \mathrm{K}\right) ;$ $\alpha_{2}=10 \mathrm{Bm} /\left(\mathrm{M}^{2} \cdot \mathrm{K}\right) ; \quad \mathrm{F}=20 \mathrm{~cm}^{2} ; \lambda_{u}=41 \cdot 10^{-3} \mathrm{Bm} /(\mathrm{M} \cdot \mathrm{K})$;

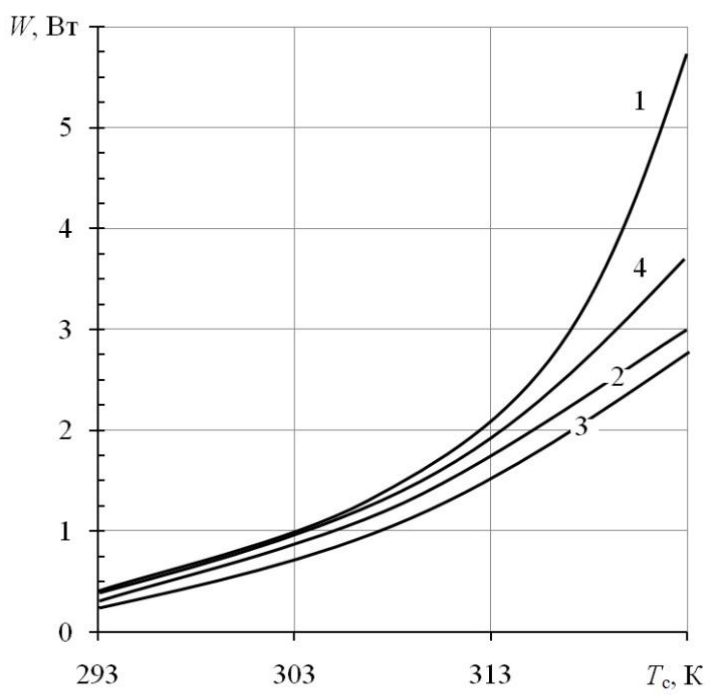

a)

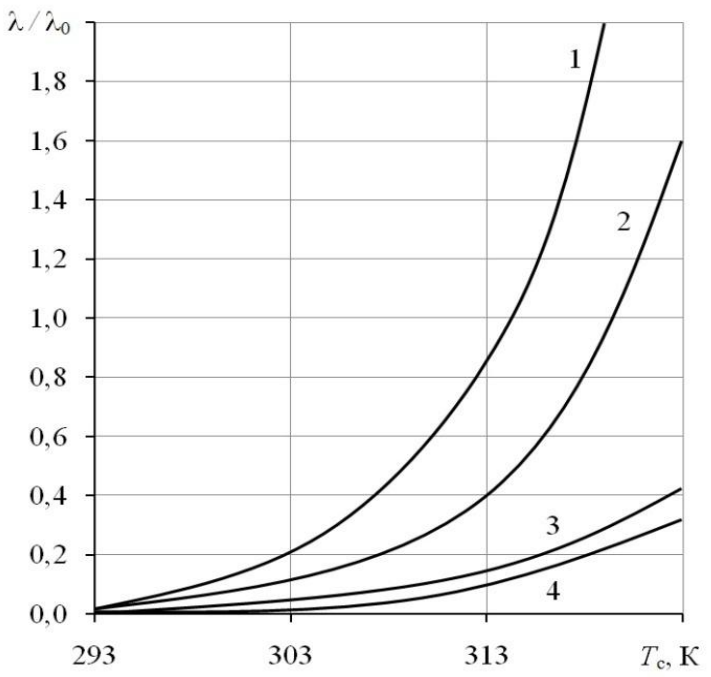

б)

Рисунок 3 - Зависимость потребляемой мощности W (a) и относительной интенсивности отказов $\lambda / \lambda_{0}$ (б) однокаскадного термоэлектрического устройства от температуры среды $T_{\varepsilon}$ при $T_{0}=293 \mathrm{~K} ; T=T_{c}+10 \mathrm{~K}$; $q_{0}=1,0 \mathrm{Bm} ; \lambda_{0}=3 \cdot 10^{-8} 1 / 4 ; \mathrm{l} / \mathrm{S}=10 \mathrm{~cm}^{-1}$ для различных начальных токовых режимов работыл: $1-Q_{0 \max }$ $(n=8,2) ; 2-\left(Q_{0} / I\right)_{\max }(n=10,6) ; 3-\left(Q_{0} / I^{2}\right)_{\max }(n=21,0)$; $4-\lambda_{\min }(n=47,1)$ 


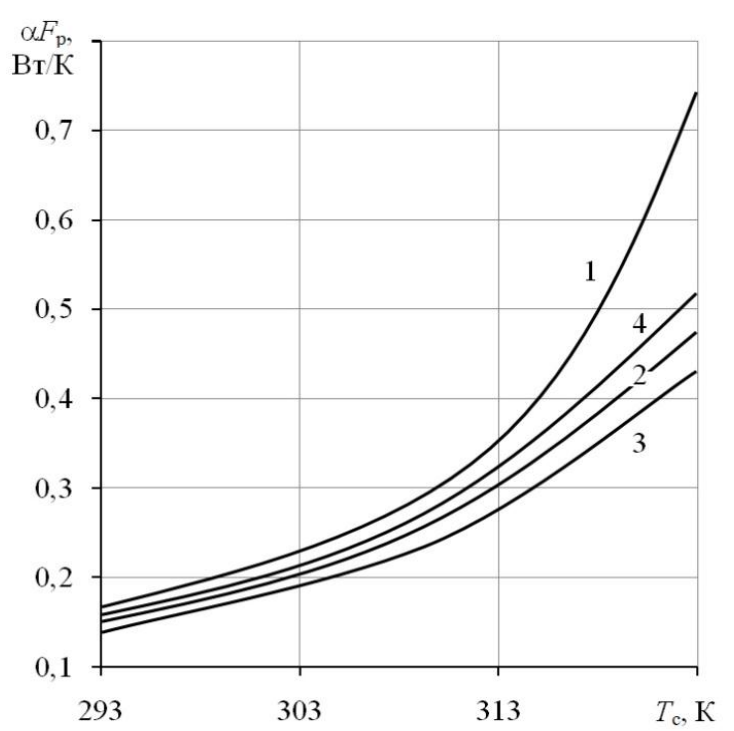

Рисунок 4 - Зависимость теплоотводящей способности радиатора $\alpha F_{p}$ однокаскадного ТЭУ от темпераmуры среды $T_{c}$ при $T_{0}=293 \mathrm{~K} ; \mathrm{T}=T_{c}+10 \mathrm{~K} ; q_{0}=$ $=1,0 \mathrm{Bm} ; l / S=10 \mathrm{~cm}^{-1}$ для различных начальных токовых режимов работы: $1-Q_{0 \max }(n=8,2) ; 2-\left(Q_{0} / I\right)_{\max }$ $(n=10,6) ; 3-\left(Q_{0} / I^{2}\right)_{\max }(n=21,0) ; 4-\lambda_{\text {min }}(n=47,1)$

Минимальная интенсивность отказов $\left(\lambda / \lambda_{0}\right)_{\min }$ обеспечивается в режиме минимума интенсивности отказов $\lambda_{\text {min }}$. Например, при $T_{\mathrm{c}}=323 \mathrm{~K}$ она составляет 0,28 , что в 27 раз меньше, чем в режиме $Q_{0 \max }$, в в 5,3 раза меньше, чем в режиме $\left(Q_{0} / I\right)_{\max }$, и в 1,4 раза меньше, чем в режиме $\left(Q_{0} / I^{2}\right)_{\max }$.

\section{4 Выводы}

Анализ результатов расчетов показал, что в качестве начального режима работы при максимальной темпера-

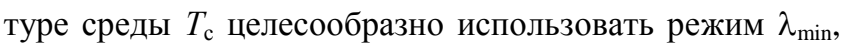
который при $T_{\mathrm{c}}=323 \mathrm{~K}$ по сравнению с режимом $\left(Q_{0} / I^{2}\right)_{\max }$ позволяет:

- уменьшить интенсивность отказов в 1,5 раза и, следовательно, увеличить вероятность безотказной работы;

- уменьшить рабочий ток в 1,33 раза;

- увеличить падение напряжения в 1,8 раз при приемлемом энергопотреблении и массогабаритных характеристиках.

\section{Литература}

1. Моисеев, В. Ф. Системы обеспечения тепловых режимов радиоэлектронной аппаратуры специального назначения [Текст] / В. Ф. Моисеев // ТРиО. - 2003. Вып. 1. - С. 43-48.

2. Зайков В. П. Прогнозирование показателей надежности термоэлектрических охлаждающих устройств. Книга 1. Однокаскадные устройства[Текст] / В. П. Зайков, Л. А. Киншова, В. Ф. Моисеев. Одесса: Политехпериодика, 2009. - 118 с.

3. Михеев М. А., Михеева И. М. Основы теплопередачи [Текст]. - Москва: Энергия, 1977. - 344 с.

Отримана в редакції 20.04.2017, прийнята до друку 06.06.2017

\section{Rational Design of Thermoelectric Cooling Device for Variable Temperature Operating Conditions}

\section{Yu. Zhuravlov}

National University «Odessa Maritime Academy», 8 Didrikhsona str., Odessa, 65029, Ukraine

The possibility of rational design of thermoelectric device in systems for providing thermal modes for radioelectronic equipment during operation in various climatic conditions is considered. The aim of the work was to find solutions that allow choosing a variant of thermoelectric device design at a changing temperature of the environment, which ensures a reduction in energy costs and an increase in reliability indicators. The calculations of the basic energy and reliability parameters of thermoelectric devices for various operating modes have been carried out. The influence of the ambient temperature is analyzed and it is shown that the heat load, operating current, power consumption and intensity of failures increase with temperature. The expediency of thermoelectric devices functioning in the mode of minimum failure rate is substantiated, which allows reducing the failure rate by 1.5 times in comparison with the maximum cooling capacity mode.

Keywords: Operating mode; Cooling capacity; Failure rate

\section{References}

1. Moiseev, V. F. (2003). Systems for providing thermal modes of radioelectronic equipment for special purposes. TRiO. Issue. 1, 43-48 (in Russian)

2. Zaykov, V. P., Kinshova, L. A., Moiseev, V. F. (2009). Prediction of reliability on thermoelectric cooling devices. B.1 Single-stage devices. Odessa: Politekhperiodika, 118. (in Russian)

3. Mikheev, M. A., Mikheeva, I. M. (1977). The fundamentals of heat transfer. Moscow: Energy, 1977, 344 (in Russian) 